\title{
Clinical Presentation and Bacterial Etiology of Adult Community Acquired Pneumonia
}

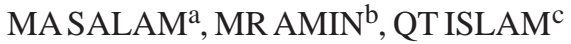

\begin{abstract}
Summary:
Introduction: Pneumonia is a worldwide, serious threat to health and an enormous socio-economic burden for health care system. According to recent WHO data, each year 3-4 million patients die from pneumonia. The clinical presentations and bacterial agents responsible for community acquired pneumonia (CAP) varies according to geography and culture.
\end{abstract}

Methods: A cross sectional observational study conducted among the 53 consecutive patients with a clinical diagnosis of CAP in admitted patient in the department of Medicine, DMCH, during January 2010 to December 2010. Hematological measurements (TC of WBC, Hb\%, ESR, platelet count), blood culture, chest $X$-ray $P / A$ view, sputum for Gram staining and culture sensitivity, sputum for AFB, blood urea and random blood sugar were done in all cases. ELISA for IgM antibody of Mycoplasma pneumoniae and Chlamydia pneumoniae were done in sputum culture negative cases.

Results: The mean $( \pm S D)$ age was $38.9 \pm 17.3$ years and Male female ratio was 3:1. Fever, chest pain and productive cough were the most common clinical features. The mean $( \pm S D)$

Introduction:

Community-acquired pneumonia (CAP) remains a common disease with high morbidity, mortality, and treatment cost. ${ }^{1}$ Criteria for the diagnosis of communityacquired pneumonia were: a history of fever with cough for $\leq 14$ days prior to admission; abnormal infiltration

a. Dr. Md. Abdus Salam, Consultant (Medicine), Shaheed Ziaur Rahman Medical College Hospital.

b. Dr. Md. Robed Amin, Associate Prof of Medicine, Dhaka Medical College.

c. Prof. Quazi Tarikul Islam, Professor of Medicine, Popular Medical College.

Address of Correspondence: Dr .Md. Robed Amin, Associate Professor of Medicine, Dhaka Medical College, Apt-C-2, House no 76, Road-5, Block-F, Bonani Dhaka, email: robedamin@yahoo.com Received: 13 August, 2014

Accepted: 24 April, 2016 respiratory rate was $23.0 \pm 2.8$ /minute. COPD and DM were found in $17.0 \%$ and $5.7 \%$ of patients respectively. Blood culture was found positive in only $1.9 \%$ of the study patients. Gram positive Cocci $62.26 \%$, Gram negative Bacilli 9.43\%, mixed Gram positive cocci and Gram negative bacilli 11.32\% and Gram negative Cocco Bacilli 1.9\% were observed and in $15.03 \%$ cases, no bacteria could be seen.

Sputum culture revealed $53.8 \%$ streptococcus pneumoniae, 26.9\% Klebsiella pneumonia as predominant organism. Mycoplasma pneumoniae and Chlamydia pneumoniae were found in $7.4 \%$ and $3.7 \%$ respectively by serological test. For Streptococcus pneumoniae, sensitive antibiotics were Amoxyclav and Levofloxacin. For Gram negative bacilli and coccobacilli, more sensitive antibiotics were Meropenem, Ceftriaxone, and Clarithromycin. The best sensitive drug were found meropenem. The mean $( \pm S D)$ duration of hospital stay was $5.0 \pm 1.7$ days with ranging from 3 to 10 days.

Conclusion: Region based bacteroiological diagnosis of Cap is important for selecting the best and sensitive drugs for complete cure.

(J Bangladesh Coll Phys Surg 2016; 34: 128-134)

shown by chest x-ray (excluding lung abscesses, bronchiectasis, and lung masses). ${ }^{2}$ Identification of the pathogen depends on isolation of the infective organism from sputum or blood. The microbial patterns reported for CAP differ considerably, depending on epidemiologic area, patient populations, and the extent and nature of the microbiologic techniques use. Knowledge of predominant microbial patterns in CAP constitutes the basis for initial decisions about empirical antimicrobial treatment. $^{3}$

According to recent WHO data, each year 3-4 million patients die from pneumonia. Pneumonia is the third most common cause of death among infectious disease in the world. ${ }^{4}$ National data on incidence, etiology and mortality of CAP is not available in Bangladesh. The incidence of CAP requiring hospitalization is estimated 
to be 258 cases per 100,000 population and 962 cases per 100,000 persons 65 years of age. ${ }^{5}$ While mortality has ranged from $2 \%$ to $30 \%$ among hospitalized patients in a variety of studies, the average rate is $14 \% .{ }^{6}$ Mortality is estimated to be $<1 \%$ for patients who are not hospitalized. ${ }^{6-7}$ The incidence of CAP is highest in the winter months.

Prospective studies for evaluating the causes of CAP in adults have failed to identify the cause of $40 \%-60 \%$ of cases of CAP, and two or more etiologies have been identified in $2 \%-5 \%$ of cases. ${ }^{5}$ The most common etiologic agent identified in virtually all studies of CAP is Streptococcus pneumoniae, and this agent accounts for approximately two thirds of all cases of bacteremic pneumonia. ${ }^{6}$ Other pathogens implicated less frequently include Haemophylus influenzae (most isolates of which are other than type B), Mycoplosma pneumoniae, Chlamydia pneumoniae, Staphylococcus aureus, Streptococcus pyogenes, Neisseria meningitidis; Moraxella catarrhalis, Klebsiella pneumoniae and other Gram-negative rods, Legionella species, Influenza virus (depending on the time of year), Respiratory syncytial virus, Adenovirus, Parainfluenza virus, and other microbes. The frequency of other etiologies, e.g., Chlamydia psittaci (psittacosis), Coxiella burnettii (Q fever), Francisella Tularenesis (tularemia) and endemic fungi (Histoplasmosis, Blastomycosis, and Coccidioidomycosis), is dependent on specific local epidemiological factors. No convincing association has been demonstrated between individual symptoms, physical findings or laboratory test results, and specific etiology. ${ }^{8}$ Even time-honored beliefs (e.g., the absence of a productive cough or lack of inflammatory sputum suggests etiologies such as species of Mycoplasma, Legionella, and Chlamydia) have not withstood close inspection. On the other hand, most comparisons have involved relatively small numbers of patients and the potential for separating causes by using constellations of symptoms and physical findings has not been evaluated.

The present study was done to describe the clinical presentation and the causative bacterial organism and their pattern of CAP in adult patients in DMCH and also the influence of patient age, previous antibiotic use, and the category of pneumonia on microbial patterns in this disease. Antimicrobial susceptibility of isolates from clinical specimens were also tested to estimate the prevalence of drug sensitivity and resistance pattern of common CAP-causing bacteria, especially Streptococcus pneumoniae and Haemophilus influenzae. Meanwhile the short term clinical outcome of antibiotic therapy for CAP was also observed.

\section{Methods:}

A Cross sectional observational study was done from January 2010 to December 2010 in the Department of Medicine, Dhaka Medical College Hospital (DMCH), in Dhaka, Bangladesh. Dhaka Medical College is situated in the center of Dhaka city. It is a tertiary care teaching and referral hospital in Bangladesh. About 27 thousands patients were treated in the Department of Medicine in 2009.

Fifty three adult patients of both sexes over 18 years of age admitted in the department of Medicine of DMCH with Fever for less than 14 days and one or more of the followings: Cough, Sputum, Haemoptysis, Pleuritic chest pain, Dyspnoea, Sign of consolidation and radiological evidence of Pneumonic consolidation were included while hospital acquired pneumonia, chemical pneumonitis ca lung, radiological evidence of fibrosis, collapse, bronchiectasis, lung abscess and tuberculosis, suspicision of immunosupression or known immunosuppressive status like HIV, Haematological or lymphoid malignancy and pt on immunosupressive drugs- steroids and chemotherapy were excluded

\section{Procedures:}

This study was approved by the ethical review committee of Dhaka Medical College.

After admission in the indoor, any suspected case of community acquired pneumonia seen by unit doctor was screened by study physician. Evaluation was made by history and physical examination in a structured case record form (CRF) by the study physician. Patients diagnosed clinically as CAP was screened in the study. Investigations were done hematologic measurements (TC of WBC, Hb\%, ESR, platelet count), blood culture, chest X-ray P/A view, sputum for Gram staining and culture sensitivity, sputum for AFB for 3 consecutive days, blood urea and random blood sugar . For scanty production of cough, patients sputum was collected after nebulization by hypertonic normal saline. Serological tests were done for the blood culture negative and sputum culture negative cases for identification of atypical organisms (e.g. Mycoplasma 
and Chlamydia pneumoniae). It was done by ELISA for IgM antibody of Mycoplasma and Chlamydia pneumoniae. Patient with positive radiological findings of consolidation was enrolled in the study. Sputum for AFB positive cases and radiology of exclusion criteria was screened out from the evaluation. The patient address (tracing) and cell number was recorded to ensure follow up. The negative sputum culture patient was also followed up as like that of culture positive cases. Antibiotic therapy of the enrolled patient was given at the discretion of the treating clinician under the supervision of respective consultant of the medicine unit. The clinical judgment of consultant was boost up by doing the CURB-65 score by the study physician.

The study used the British Thoracic Society of severe community acquired pneumonia detected by CURB-65 which includes presence of any of the following:

0. Confusion

1. Urea $7 \mathrm{mmol} / \mathrm{l}$

2. Respiratory rate 30 breath $/ \mathrm{min}$

3. Systolic blood pressure $<90 \mathrm{~mm}$ of $\mathrm{Hg}$ or Diastolic blood pressure $<60 \mathrm{~mm}$ of $\mathrm{Hg}$

4. Age65 years

1 point was scored for each feature present. On the basis of points treatment protocol was adjusted.

During treatment, oral temperature was recorded and frequently physical examinations were performed up to discharge. Patients were supplied a structured questionnaire regarding changes in symptomatology and general well being. Patients were asked to report 2 weeks after completion of treatment for follow up. Any patient who failed to come for follow up in time was called upon by cell phone (contact number of patient). Thus a short term outcome after the treatment was observed.

\section{Microbiological evaluation}

Sputum samples were collected from all patients enrolled in the study. Representative sputum originated from the lower respiratory tract was defined as that containing $>25$ granulocytes and $<10$ epithelial cells per low power field microscopic view. Validated sputum was cultured in blood agar, chocolate agar and McConkey's agar media. Isolation and identification of microorganism was done according to the standard method.
Blood samples (6-8 ml) were collected aseptically from patients for blood culture and serological test. Serum was separated and stored at $-4^{\circ} \mathrm{C}$. Primary blood culture was done in Trypticase soya broth and secondary blood culture was done on blood agar, chocholate agar and McConkey's agar media.

\section{Antimicrobial susceptibility testing:}

Antimicrobial susceptibility was determined by the disc diffusion method of modified Kirby-Bauer (1966) technique using Blood agar media (for Streptococcus pneuminiae), Mueller-Hinton agar media (for Escherichia coli, Klebsiella pneumoniae and Pseudomonas species) and Chocolate agar media (for Haemophylus influenzae) and antimicrobial discs (Oxoid, UK) . Following antimicrobials and their concentration per disc were used for susceptibility tests:

5. For Gram positive cocci : Amoxyclav (30 microgram), Levofloxacin (5 microgram), Azithromicin (15 microgram), Cefixime (30 microgram) and Doxycycline (30 microgram).

b) For Gram negative bacilli and coccobacilli : Meropenem

(10 microgram), Ceftriaxone (30 microgram), Amikacin

(10 microgram), Clarithromycin (5 microgram), Ciprofloxacin

(5 microgram), Amoxyclav (30 microgram) and Cefixime

(30 microgram).

Methods of susceptibility testing: The agar plates were dried in an incubator at $37^{\circ} \mathrm{C}$ for 30 minutes before use. With a sterile wire loop, pure and isolated colonies were picked up and was suspended into a sterile tube containing $2 \mathrm{ml}$ of normal saline. The turbidity of the inoculum was standardized to the equivalent to that of 0.5 of Mac Farland standard. A sterile cotton swab was immersed into the bacterial suspension and the excess suspension was removed by rotating the swab with a firm pressure against the inner side of the tube above the fluid level. The swab was then streaked evenly on the entire surface of the concerned plate. The discs were then placed on the inoculum surface by a sterile forcep $15 \mathrm{~mm}$ away from the edge of the petridish with $25 \mathrm{~mm}$ gap in between the discs. All plates were 
incubated at $37{ }^{0} \mathrm{C}$ - aerobically for Blood agar and Mueller-Hinton agar and microaerobically ( by candle jar) for Chocolate agar medium. Measurement of inhibition zone: standard procedure was followed. ${ }^{9}$

\section{Data analysis:}

Categorical data was presented as frequency and percentage and continuous variable presented as mean and standard deviation. All data was analyzed by SPSS (Statistical Package for Social Science) 16 windows version.

Results:

This prospectively study enrolled 53 cases of CAP fulfilling the eligibility check list. The various characteristics of the cases are presented in table 1 . The mean $( \pm \mathrm{SD}$ ) age was $38.9 \pm 17.3$ years with ranged from 18 to 90 years and maximum number (24.5\%) of patients was found in the age group of $31-40$ years.

Fever was present in all of the study patients. Chest pain 43(81.1\%) and copious productive cough 34(64.2\%) were present in the study patients. During physical examination most of the patients had $100^{0}-102^{0} \mathrm{~F}$ temperature (71.7\%) and all patients had consolidation. (Table II)

CURB-65 Pneumonia severity scoring revealed 48 (90.6\%) had $0-1,5$ (9.4\%) had 2 and none were found to have 3 while the mean $( \pm S D)$ respiratory rate was $23.0 \pm 2.8$ /minute with range from 18 to 32 /minute. it was observed that COPD and DM were found 9 (17.0\%) and $3(5.7 \%)$ respectively in study patients. The mean $( \pm \mathrm{SD})$ TC was $14804 \pm 3533 /$ cumm, Neutrophil $78.5 \pm 7.1 \%$, Lymphocyte 17.0 $\pm 6.7 \%$, Monocyte 2.6 $\pm 1.4 \%$, Eosinophil $2.3 \pm 1.2 \%$, Basophil $0.0 \pm 0.0 \%$ and ESR $57.6 \pm 13 \mathrm{~mm}$. In blood culture Pseudomonas species was found in 1 (1.9\%) of the study patients. This patient (42 yrs female) had known diabetes mellitus and presented with high continued fever and copious productive sputum for five days. At the time admission random blood sugar was 15 $\mathrm{mmol} / \mathrm{L}$. The hospitalization of this patient was 10 days and she received meropenem with slow but complete recovery.

In sputum tested by Gram stain, only Gram positive cocci were seen in 33 (62.26\%) samples, only Gram negative bacilli in 5 (9.43\%) samples. Mixed Gram positive cocci and Gram negative bacilli in 6 (11.32\%) samples, only one sample (1.88\%) showed presence of
Gram negative coccobacilli. Bacteria could not be found in $8(15.03 \%)$ samples. (Table III)

Sputum culture was done in all patients and 26 (49.1\%) positive growth out of which 5 cases used antibiotics before enrolment. Tweenty seven (50.9\%) were found no growth where 20 cases used prior antibiotics and 7 cases did not use prior antibiotics before hospitalization. The use of antibiotics were variable in duration in positive and negative growth cases.

Streptococcus pneumoniae was identified as the sputum culture positive in 14 / 26 cases. These 14 cases are also out of 33 Gram stain positive cocci (Not shown in table). Klebsiella pneumoniae was found 7 (26.9\%) as the common isolates among Gram negative cases.(Table IV)

In case of Streptococcus pneumoniae, Amoxyclav showed the highest sensitivity (78.6\%), followed by Levofloxacin (64.3\%). (Table V)

Most of the given antibiotics were amoxyclav (34.6\%), clarithromycin (30.8\%), Ceftriaxone (30.8\%) and Meropenem (23.1\%).(table VI)

Mycoplasma pneumoniae and Chlamydia pneumoniae were found in $2(7.4 \%)$ and 1 (3.7\%) respectively according to serological test of the study patients. The mean $( \pm \mathrm{SD}$ ) duration of hospital stay was $5.0 \pm 1.7$ days with ranged from 3 to 10 days.

\section{Discussion:}

In this small series of patients with Community Acquired Pneumonia (CAP), microorganism could be identified in $49 \%$ cases in sputum culture and Streptococcus pneumoniae was the most frequent organism(14/26) resulting in classical clinical features supporting the statement that Streptococcus pneumoniae was the commonest organism of community acquired pneumonia (CAP). ${ }^{10}$

Berntsson et al. ${ }^{11}$ observed Streptococcus pneumoniae $49.0 \%$ in their study. Woodhead et al. ${ }^{12}$ found Streptococcus pneumoniae $36.0 \%$ and Escherichia coli $1.0 \%$. Fang et al. ${ }^{8}$ observed that Streptococcus pneumoniae in $15.3 \%$, Haemophylus influenzae in $10.9 \%$ in his study. Sullivan et al. ${ }^{13}$ found Klebsiella pneumoniae 2.0\% and Escherichia coli $4.0 \%$. So it is observed that there are variable percentage in different studies especially for Streptococcus pneumoniae. In this present study about fifty percents sputum culture were negative which might be due to other aetiological 
agents e.g. viral or Legionella pneumophila or use of antibiotic prior to hospitalization. Mamun et al. ${ }^{14}$ showed that one fifth of the patients used antibiotics by dispensing practices in rural Bangladesh. The frequency of Streptococcus pneumoniae in present study is like that of similar observations showed Streptococcus pneumoniae 20.9\%, Klebsiella pneumoniae $4.7 \%$ and Staphylococcus aureus $2.3 \%$ in non diabetic patients. ${ }^{6,11,15,17,18}$

Gram positive Cocci in staining procedure was found among more than half of enrolled patients. Fang et al. ${ }^{8}$ observed $14.8 \%$ Gram positive cocci in their study, which is less than the present study. Gram stain has been found a useful test and reliable for targeting pathogen-directed first-line antibiotic therapy in CAP patients. Moderate to large amounts of Gram-positive diplococci was seen in sputum Gram stain of purulent sputum observed by Stralin ${ }^{19}$ which is consistent with the present study. Macfarlane et al. ${ }^{15}$ and Andrews et al. ${ }^{16}$ observed Pseudomonas species $11.0 \%$ and $2.0 \%$ respectively in their study patients. Only one patient of this present study showed Pseudomonas species as it was observed in blood culture. Stralin ${ }^{19}$ observed Pseudomonas species as the rare cause of CAP in his study which is also consistent with the present one.

In this personal series sensitivity pattern of isolated strain of bacteria from CAP patients is alarming that resistant bacteria is emerging. It was observed from this study that isolated Klebsiella strain was mostly resistant to commonly used antibiotics for CAP. Other isolated organisms like Pseudomonas, Escherichia coli, were also resistant to $B$-lactamase inhibitor, Macrolides and third generation cephalosporin. Streptococcus pneumoniae were sensitive to commonly used antibiotic for CAP. This study also revealed Meropenem were the most effective antibiotic for CAP. These antiobiotics are costly and not recommended by the guideline published by American thoracic society (2004) and Infectious disease society of America (IDSA 2004).

Frequently use $B$-lactam antiobiotic and Macrolides for the treatment CAP are first line regimens but emerging strain are more resistant to these conventional antibiotics. Multi drug resistant to B-lactamase, Macrolides and Fluroquinolone is an emerging problem and complicating the management of CAP ${ }^{20}$. In a study ICDDRB Rahman et al. ${ }^{21}$ found Pneumococcus serotype is resistant to penicillin and macrolides posing threat to Bangladesh and some other Asian countries. Saibal ${ }^{18}$ showed the sensitivity pattern against the Streptococcus pneumoniae to Amoxyclav 83\%, and Klebsiella pneumoniae to Ceftriaxone $73.3 \%$ which is comparable with the present study.

The antibiotics chosen by physicians were according to preference Amoxyclav, Clarithromycin, Ceftriaxone and Meropenem. Woodhead et al. ${ }^{12}$ showed almost similar given antibiotics in their study patients.

Mycoplasma pneumoniae and Chlamydia pneumoniae were found by serological test in this study patients. Sullivan et al. ${ }^{13}$, Macfarlane et al. ${ }^{14}$ and Ngeow et al. ${ }^{4}$ observed Mycoplasma pneumoniae 9.3\%, 2.4\% and $9.2 \%$ respectively in their study. Falguera ${ }^{17}$ observed Chlamydia pneumoniae $11.0 \%$, Mycoplasma pneumoniae $9.0 \%$. Sohn et al. ${ }^{22}$ showed Mycoplasma pneumoniae $4.8 \%$ and Chlamydia pneumoniae 4.0\%, which are comparable with the current study.

In this present study, patients mean age and age range is similar to different studies. Fang et al. ${ }^{8}$ had shown in their series, the age ranged of their patients were 23 to 92 years, which closely agrees with the present study. Liapikou et al. ${ }^{23}$ and Sohn et al. ${ }^{22}$ observed higher mean age in their study, where the mean age were $68.9 \pm 17.9$ years and $54.6 \pm 17.8$ years respectively. This variation in their study may be due to higher life expectancy in their population.

Liapikou et al. ${ }^{23}$ study, where the authors found male female ratio was almost 2:1 while in this personal series it was observed that more than three fourths of the patients was male which is comparable. However, Fang et al. ${ }^{8}$ and Sohn et al. ${ }^{22}$ observed male female ratio was almost 1:1. Male patients were higher in this study, which may be due to access to health care facility by male is easier than female as she had to maintain the family and children.

The frequency of fever, chest pain and cough in this series was common feature. Almost similar observations regarding the clinical presentations were also found by Andrews et al. ${ }^{16}$, Fang et al. ${ }^{8}$, Sohn et al. ${ }^{22}$ and Ngeow et al. ${ }^{4}$. Ashraf et al. ${ }^{24}$ showed fever and cough were $89.0 \%$ and $99.0 \%$ respectively in their study children.

All patients had documented temperature during hospital stay. In a study of pediatric population of same 
country Ashraf et al. ${ }^{24}$ showed $44.0 \%$ had $>102^{0} \mathrm{~F}$ temperature among the study children. Fang et al. ${ }^{8}$ observed in their study that increasing age was associated with lower temperature. Sign of consolidation were found in all cases in this series. Sohn et al. ${ }^{22}$ found consolidation in $87.5 \%$. Almost similar findings obtained by Andrews et al. ${ }^{16}$ and Liapikou et al. ${ }^{23}$. Majority of the findings are similar with the present study regarding this physical sign.

Prognosis of the patient was seen in hospitalized patient through CURB score. More than 90 percent in this study is within score 1 . Liapikou et al. ${ }^{23}$ observed almost similar findings regarding the CURB-65 scoring in their study. CURB-65 score was not similar with Schuetz et al. ${ }^{25}$ study, where the authors observed $1-2$ score in $0.2 \%$ of their study patients possibly because the study was done in critical care unit. Saiba ${ }^{18}$ showed CURB-65 scoring $0-1$ in $62.8 \%$ and 2 in $16.3 \%$ in non diabetic patients. CAP cases in present study were not very serious to be shifted to ICU and mortality was also absent. As the sample size is small, the actual poor prognostic factors may not be reflected here.

The respiratory rate range was similar to Schuetz et al. ${ }^{25}$ who observed respiratory rate ranged from 16 to $25 / \mathrm{min}$ in their study and is also corresponds with the good outcome to the patients. .

Concomitant diseases of COPD and DM were observed in the study. Fang et al. ${ }^{8}$ observed COPD in $31.4 \%$ and DM in $13.4 \%$ in their study which was little higher frequency than present study. Berntsson et al. ${ }^{11}$ found DM 4.0\%, which is similar with the current study frequency. Flguera ${ }^{17}$ observed $16.7 \%$ patients had DM. Liapikou et al. ${ }^{23}$ observed COPD 37.9\%, 19.4\% and $45.0 \%$ respectively. Similarly, Liapikou et al..$^{23}$ observed $19.0 \%$ patient had DM in the study. Although random blood sugar was taken as a screening criteria in this study for DM, it may turn up to be different if Fasting or OGTT should have been undertaken.

The mean duration of hospital stay was similar to few studies. Falguera ${ }^{17}$ and Liapikou et al. ${ }^{23}$ showed the mean duration of hospital stay was $5 \pm 1.2$ days and $6 \pm 1.9$ days which was similar in this study. Saibal ${ }^{18}$ found the mean duration of hospital stay was $7.7 \pm 1.7$ days in non diabetic patients. Ashraf et al. ${ }^{24}$ found duration of the clinic stay $>10$ days was $5.0 \%$ of the study children.
Limitation of the study: This descriptive study is a study with small sample and requires further studies from different levels of hospitals. A nationally representative surveillance system for CAP could replace periodic small studies.

\section{Conclusion:}

Streptococcus pneumoniae was common organism for CAP identified by sputum culture. Mycoplasma pneumoniae and Chlamydia pneumoniae were found by serological test. For CAP associated with Streptococcus pneumoniae, sensitivity results were in favour of Amoxyclav and Levofloxacin. For CAP associated with Gram negative bacilli and coccobacilli, sensitivity status were in favour of Meropenem, Ceftriaxone and Clarithromycin respectively. Common use of antibiotics in community can lead to difficulty in identifying organism was observed in this study.

Conflicts of interest: None declared

\section{References:}

1. Hirani NA., Macfarlane JT. Impact of management guidelines on the outcome of severe Community Acquired Pneumonia. Thorax 1997; 52: 17-21.

2. Bartlett JG, Breiman RF, Mandell LA. Community-acquired pneumonia in adults:guidelines for management. The infectious Diseases Society of America, Clinical Infectious Diseases 1998; 26:811-838.

3. Woodhead M, Blasi F, Ewig S, Huchon G, leven M, Ortqvist A. European Respiratory Society; European Society of Clinical Microbiology and Infectious Diseases. Guidelines for the management of adult lower respiratory tract infections. European Respiratory Journal 2005;26: pp.1138-1180.

4. Ngeow YF, Suwanjuthab S, Chantarojanasririb T, Wangc F, Sanield M, Alejandriad M. An Asian study on the prevalence of atypical respiratory pathogens in Community Acquired Pneumonia. International Journal of Infectious Diseases 2005;(9):144-153.

5. Marston BJ, Plouffe JF, File TM. Incidence of communityacquired pneumonia requiring hospitalizations: results of a population-based active surveillance study in Ohio. Arch Intern Med 1997;157:150--160.

6. Fine MJ, Smith MA, Carson CA 1996. Prognosis and outcomes of patients with community-acquired pneumonia. JAMA 1996;275:134-41.

7. Fine MJ, Auble TE, Yealy OM. A prediction rule to identity low-risk patients with Community Acquired Pneumonia, New England Journal of Medicine 1997; 336:243-50. 
8. Fang GD, Fine M, Orloff. New and emerging etiologies for Community Acquired Pneumonia with implications for therapy; a pro-spective multicenter study of 359 cases. Medicine (Baltimore) 1990; 69:307-16.

9. Washington C. Legionella and Legionnaires' disease : a review with emphasis on environmental studies and laboratory diganosis. Crit Rev Clin Lab Sci 1985;21(4):323-81.

10. Mandell LA, Wunderink RG, Anzueto A, Barlett JG, Campbell GD, Dean Whitney CG. Infectious Diseases Society of American and American Thoracic Society. Clinical Infectious Diseases 2007;44(2):pp.27-72.

11. Berntsson E, Blomberg J, Lagergrd T, Trollfors B. Etiology of Community Acquired Pneumonia in Patients Requiring Hospitalization. European Journal of Clinical Microbiology 1985;4(3):268-272.

12. Woodhead MA, Macfarlane JT, McCracken JS, Rose DH, Finch RG, 'Prospective study of the aetiology and outcome of pneumonia in the community. The Lancet 1987;1:671-4.

13. Sullivan RJ, Dowdle WR, Marine WM, Hierholzer JC. Adult pneumonia in a general hospital. Archives of Internal Medicine 1972;129,935-42.

14. Mamun KZ, Tabassum S, Hussain MA, Shears P, Hart CA. A survey of antimicrobial prescribing and dispensing practices in rural Bangladesh. Mymensingh Medical Journal 2006;15,81-84

15. Macfarlane JT, Miller AC, Smith WHO. Comparative radiographic features of Community Acquired Legionnaires disease; Pneumococcal pneumoniae, Mycoplasma pneumoniae and psittacosis. Thorax 1984; 39:28-33.

16. Andrews BE, Bartlett CLR, Ellis DA, Farr BM, Gooch CD et al. A Survey of Aetiology, Mortality, Prognostic Factors And Outcome Medicine. New Series 1987;62(239):195-220.

17. Falguera M, Pifarre R, Martin A, Sheikh A, Moreno A. Etiology and outcome of Community Acquired Pneumonia in patients with diabetes mellitus. Chest 2005;128:5.
18. Saibal AA. Community Acquired Pneumonia: Comparison between Diabetic and Non Diabetic hospitalized patients. Thesis of Dhaka University (Medicine Faculty) 2010.

19. Stralin K. Usefulness of aetiological tests for guiding antibiotic therapy in Community Acquired Pneumonia. International Journal of Antimicrobial Agents 2008; 31:3-11.

20. Shah PB, James C. Giudice, Russell Griesback. Jr, Thomas F. Morley, Amita Vasoya. The newer guidelines for the Management of Community-acquired pneumonia. Journal of infectious diseases 2004; 104:521-26.

21. Rahman M, Hossain S, Shoma S and Rashid H. Emergence of a Unique Multiply-Antibiotic-Resistant Streptococcus pneumoniae Serotype 7B Clone in Dhaka, Bangladesh. Journal of clinical microbiology 2006;44 (2):4625-4627.

22. Sohn JW, Park SC, Choi YH, Woo HJ, Chjo YK, Lee JS et al. Atypical pathogens as etiologic agents in Hospitalized patients with Community Acquired Pneumonia in korea: A prospective multi-center study. Journal of Korean Medical Science 2006;21:602-7.

23. Liapikou A, Ferrer M, Polverino E, Balasso V, Esperatti M, Piner R et al. Severe Community Acquired Pneumonia: Validation of the Infectious Disease Society of America/ American Thoracic Society Guidelines to predict and Intensive Care Unit Admission. Clinical Infectious Disease 2009;48:377-85.

24. Ashraf H, Jahan SA, Alam NH, Mahmud R, Kamal SM. Day-care management of severe and very severe pneumonia, without associated co-morbidities such as severe malnutrition, in an urban health clinic in Dhaka, Bangladesh. Archives Diseases of Children 2008;93: 490-494.

25. Schuetz P, Wolbers M, Christ-Crain M, Thomann R, Falconnier C, Widmer I. Prohormones for prediction of adverse medical coutcome in Community Acquired Pneumonia and lower respiratory tract infections. Critical Care 2010;14:106. 